\title{
CLASSIFICATION OF BUDGET-INVARIANT MONOTONIC PREFERENCES
}

\author{
Walter TROCKEL \\ University of California, San Diego, CA 92093, USA, and Bielefeld University, 4800 Bielefeld, FRG
}

Received 15 December 1988

Accepted 21 February 1989

\begin{abstract}
A linear utility representation theorem due to Blackwell and Girshick (1953) is used to answer the question as formulated by Grandmont (1987) whether Cobb-Douglas representable preferences are the only ones which are budget-invariant.
\end{abstract}

\section{Introduction}

A main difficulty in deriving an aggregate demand function which is sufficiently structured as to meet the requirements of general equilibrium theory consists in adequately modelling the idea of suitable dispersion for distributions on a space of preferences. This problem has been satisfactorily solved in the context of 'Smoothing Demand by Aggregation' by Dierker, Dierker, and Trockel (1984). A special case of their concept of price- (or budget-) dispersion has been used by Grandmont (1987) to derive a version of a theorem due to Hildenbrand (1988) on the law of demand, which is based, however, on assumptions of preference distributions rather than on income distributions.

The usefulness of the concept of budget-dispersed preference distributions depends crucially on the non-degeneracy of orbits of preferences generated from given preferences via the action of the group of budget variations.

It has been remarked by Grandmont (1987) that Cobb-Douglas representable preferences are budget-invariant, and therefore, their orbits are totally degenerate, i.e., singletons their only elements being the original preferences. From the point of view of structuring demand, this is not bad news, however, since all the potential structure we would like to derive for aggregate demand is already present on the individual level in the case of Cobb-Douglas representable preferences.

So we are left with the question whether Cobb-Douglas representable preferences are the only bad ones.

The answer is affirmative for monotonic continuous preferences and can be directly derived from suitable versions of the linear utility representation theorems as, for instance, Theorem 4.3.1 in Blackwell and Girshick (1953) or Theorem 8 in Herstein and Milnor (1953).

I shall summarize the first mentioned of these results together with a subsequent remark in a lemma from which I shall then derive the classification result for budget-invariant monotonic continuous preferences. 


\section{The Linear Utility Representation Lemma}

Let $R$ be a preference on $\mathbb{R}^{\prime}, l \in \mathbb{N}$, i.e., a complete transitive binary relation on $\mathbb{R}^{l} . R$ is representable if there is a real-valued function $u$ on $\mathbb{R}^{l}$ such that

$\forall x, y \in \mathbb{R}^{\prime}, x R y \Leftrightarrow u(x) \geqslant u(y)$

$R$ is trivial iff it is representable by a constant function. $R$ is upper-semicontinuous iff for any $x \in \mathbb{R}^{l}$ the set $R^{-1}(x):=\left\{y \in \mathbb{R}^{l} \mid y R x\right\}$ is closed. $R$ is weakly monotonic iff for any $x, y \in \mathbb{R}^{\prime}$ we have

$\left[x-y \in \mathbb{R}_{+}^{\prime}\right] \Rightarrow[x R y]$.

Finally, $R$ is translation-invariant iff for any $x, y, z \in \mathbb{R}^{l}$

$x R y \Leftrightarrow x+z R y+z$.

Then Theorem 4.3.1 and subsequent remarks in Blackwell and Girshick (1953) yield the following:

Lemma. A non-trivial weakly monotonic preference on $\mathbb{R}^{\prime}$ is representable by a non-negative linear function iff it is upper semi-continuous and translation-invariant.

\section{Application to budget-invariance}

Let $\succsim$ be a preference on the strictly positive conc, $\mathbb{R}_{++}^{l}$, of the commodity space, $\mathbb{R}^{\prime}, l \in \mathbb{N}$.

The space of budgets (or, equivalently, if wealth is fixed, of non-normalized price-systems) is also modelled as $\mathbb{R}_{++}^{\prime}$.

Any slight or major variation of a price-system $p=\left(p_{1}, \ldots, p_{l}\right) \in \mathbb{R}_{++}^{\prime}$ resulting in $p^{\prime}=$ $\left(p_{1}^{\prime}, \ldots, p_{l}^{\prime}\right) \in \mathbb{R}_{++}^{\prime}$ can be effected as $q * p=q^{\prime}$, i.e., by coordinate-wise multiplication. Therefore, the group $\left(\mathbb{R}_{++}^{l}, *\right)$ can be looked at as the group of budget- or price variations, or, equivalently, of budgets or prices, acting on the price space $\mathbb{R}_{++}^{l}$, i.e., on itself.

Similarly, $\left(\mathbb{R}_{++}^{l}, *\right)$ acts on the commodity space $\mathbb{R}^{l}$. This latter action, moreover, induces an action of $\left(\mathbb{R}_{++}^{l}, *\right)$ on any space $P$ of preferences on $\mathbb{R}_{++}^{l}$ in the following way:

$a: \mathbb{R}_{++}^{l} \times P \rightarrow P:(q, \succsim) \rightarrow \succsim_{q}$,

where $\succsim_{q}$ is defined by

$q * x \succsim_{q} q * y \Leftrightarrow x \succsim y$.

I abbreviate $q * x$ by $x^{q}$.

Now, a preference $\succsim$ on $\mathbb{R}_{++}^{l}$ is budget-invariant iff $\forall q \in \mathbb{R}_{++}^{l}: \succsim_{q} \equiv \succsim$.

Next, I shall exploit that budget-invariant preferences on $\mathbb{R}_{++}^{I}$ correspond in a one-to-one way to translation-invariant preferences on $\mathbb{R}^{l}$. Indeed, look at the topological group isomorphisms,

$L:\left(\mathbb{R}_{++}^{\prime}, *\right) \rightarrow\left(\mathbb{R}^{l},+\right):\left(x_{1}, \ldots, x_{l}\right) \rightarrow\left(\ln x_{1}, \ldots, \ln x_{n}\right)$

and

$E:\left(\mathbb{R}^{l},+\right) \rightarrow\left(\mathbb{R}_{++}^{\prime}, *\right):\left(x_{1}, \ldots, x_{l}\right) \rightarrow\left(\exp x_{1}, \ldots, \exp x_{l}\right)$. 
Not only preserves the isomorphism $L$ the vector ordering of $\mathbb{R}^{l}$ and, hence, all kinds of monotonicity, it also induces a bijection $L^{*}$ from $P$ onto some space $L^{*}(P)$ of preferences on $\mathbb{R}^{l}$, i.e., $\succsim \rightarrow L^{*}(\succsim)$, via

$x \succeq y \Leftrightarrow L(x) L^{*}(\succsim) L(y)$.

Clearly, $R:=L^{*}(\succsim)$ is translation-invariant if and only if $\succsim$ is budget-invariant. Also $R$ is weakly monotonic iff $\succsim$ is so. To apply our above lemma, we just have to look at what happens to hyperplanes in $\mathbb{R}^{\prime}$ under the map $E$.

Consider a typical hyperplane

$H_{p, c}:=\left\{x \in \mathbb{R}^{l} \mid\langle p, x\rangle=c\right\}, \quad p \in \mathbb{R}^{l} \mid\{0\}, c \in \mathbb{R}$,

where $\langle\cdot, \cdot\rangle$ denotes the standard inner product of $\mathbb{R}^{l}$. We get (with $\bar{c}:=\exp c$ ),

$E\left(H_{p, c}\right)=\left\{x \in \mathbb{R}_{++}^{l} \mid\left\langle p_{1}\left(\ln x_{1}, \ldots, \ln x_{l}\right)\right\rangle=c\right\}=\left\{x \in \mathbb{R}_{++}^{l} \mid x_{1}^{p_{1}} \ldots x_{l}^{p_{l}}=\bar{c}\right\}$.

Note that in general there are budget invariant preferences which do not even generate demand correspondences. Take, for instance, $l=2, p_{1}=1 / 3, p_{2}=-2 / 3$. Then a typical indifference curve of a budget-invariant preference in $\mathbb{R}_{++}^{1}$ has the following form:

$\left\{x \in \mathbb{R}_{++}^{2} \mid x_{1}=\bar{c} x_{2}^{2}\right\}, \quad \bar{c} \in \mathbb{R}_{++}$.

If the representing utility function increases with $\bar{c}$ then the preference is strictly convex, but it fails to have a maximal element in the budget set $\left\{x \in \mathbb{R}_{++}^{2} \mid\left\langle p_{1} x\right\rangle \leqslant w\right\}$ for any $p \in \mathbb{R}_{++}^{2}$ and $w \in \mathbb{R}_{++}$.

Considering the formula for $E\left(H_{p, c}\right)$ we see that it is a Cobb-Douglas indifference set iff $\rho \in \mathbb{R}_{++}^{l}$. But this is the case iff $H_{p, c}$ has its normal $p$ pointing to the strictly positive cone $\mathbb{R}_{++}^{l}$. Accordingly, under monotonicity assumptions the continuous budget-invariant preferences are exactly the Cobb-Douglas representable ones. Formally:

Proposition. Let $\succsim$ be a non-trivial, upper-semicontinuous, budget-invariant weakly monotonic preference on $\mathbb{R}_{++}^{l}$. Then

$\exists p \in \mathbb{R}_{+} \mid\{0\} \forall x, y \in \mathbb{R}_{++}^{I}:[x>y] \Leftrightarrow\left[x_{1}^{p_{1}} \ldots x_{l}^{p_{l}}>y_{1}^{p_{1}} \ldots y_{l}^{p_{l}}\right]$.

Moreover, $p \in \mathbb{R}_{++}^{l}$ iff $\succeq$ is strongly monotonic, i.e., $\left[x-y \in \mathbb{R}_{+}^{l} \mid\{0\}\right] \Rightarrow[x \succ y]$.

Note that, obviously, also the converse holds true, i.e., every $p \in \mathbb{R}_{+}^{l} \mid\{0\}$ represents a continuous, weakly monotonic budget-invariant preference.

So we have established that for (weakly) monotonic continuous preferences budget-invariance and Cobb-Douglas representability are equivalent.

Our result overlaps with one due to H. Dierker (1986). She states that among all homogeneous demand functions satisfying Walras' Law, the only ones which are invariant under the action of the 
group of budget-variations are those induced by Cobb-Douglas utility functions. Her specific context of demand functions covers even those which are not derivable from individual utility maximization.

On the other hand, H. Dierker's result does not provide an answer for non-convex preferences, for convex ones whose induced demand fails to satisfy Walras' Law and for those for which there does not even exist a demand correspondence.

\section{References}

Blackwell, D. and M.A. Girshick, 1953, Theory of games and statistical decisions (Wiley, New York).

Dierker, E., H. Dierker and W. Trockel, 1984, Price-dispersed preferences and $C^{1}$ mean demand, Journal of Mathematical Economics 13, 11-42.

Dierker, H., 1986, Existence of Nash equilibrium in pure strategies in an oligopoly with price setting firms, Discussion Paper no. A-37, SFB303 (University of Bonn, Bonn).

Grandmont, J.M., 1987, Distribution of preferences and the 'law of demand', Econometrica 55, 155-161.

Herstein, I.N. and J. Milnor, 1953, An axiomatic approach to measurable utility, Econometrica 21, 291-297.

Hildenbrand, W., 1988, On the 'law of demand', Econometrica 51, 997-1019. 